\title{
Prevalence, Sources and Severity of Stress among New Undergraduate Medical Entrants
}

\author{
Jain, $S .^{1}$, Jain, $P .^{2}$, Jain, A.K. ${ }^{3}$
}

\begin{abstract}
Objectives: The past decade has seen a globally increasing trend in the prevalence of stress among medical students. The objectives of our study were to assess stress prevalence, sources of stress and their severity and to assess the determinants of stressed cases in new undergraduate medical entrants.

Methods: Two hundred and fourteen students ( $M=130, F=84)$, who had just completed their first week in the institute, participated voluntarily in a cross-sectional, self-administered questionnaire based study. After collecting demographic data, the prevalence of stress was assessed using the 12 item General Health Questionnaire (GHQ-12) and sources of stress and their severity by the 40 item Medical Students Stressor Questionnaire (MSSQ).
\end{abstract}

Results: Demographic characteristics of male and female participants were comparable. Prevalence of stress was $42.1 \%(\mathrm{M}=27 \% \mathrm{~F}=17 \%)$ of which almost $8 \%$ had severe stress. The severity of academically related stressors was found to be the highest among medical students, with approximately one-third $(34.1 \%)$ of the participants perceiving them to be causing high or severe stress. Self rated severity of all other stressor domains was either mild or moderate in more than $85 \%$ of the participants. There was a significant correlation of GHQ with total MSSQ scores $(r=0.48, p<0.01)$, as well as all six stressor domains $(r=0.22$ to $0.53, p<0.01)$. By logistic regression analysis stressed cases were associated with group activity related stressors $(B=1.095, S E=0.31$, Wald $=12.39, p<0.001)$.

Conclusion: Considerably high prevalence of stress was self-reported by new medical undergraduate entrants. Academic related factors were greater sources of stress when compared to non-academic factors in these participants.

Key words: GHQ, Medical students, MSSQ, Stress, Stressors

\section{Introduction}

Stress has been defined as anything that disrupts the physical or psychological wellbeing of a normal individual. It occurs due to the inability of the individual to cope up with the demands and pressure of the situation (Michie, 2002). Any stimulus which evokes a stress response is termed as a stressor (Lazarus, 1990). Stressors may be real or imagined, and endogenous or exogenous factors or events.

${ }^{1}$ Assistant Professor

NDMC Medical College and Hindu Rao Hospital,

New Delhi, India.

${ }^{2}$ Associate Professor

${ }^{3}$ Director and Professor

Department of Physiology, Maulana Azad Medical College, New Delhi, India.

Corresponding Author:

Shikha Jain, CG-3,606, Supertech Capetown,

Noida, Sector-74, Uttar-Pradesh-201301, India

Email:jshikha23@yahoo.in
The overall impact of a stressor depends upon its features and the characteristics of the affected individuals (Brown et al., 2009).

The past decade has seen a globally increasing trend in the prevalence of stress among the medical and healthcare professionals (Mohsin et al., 2010; Muhamad et al., 2010; Trushna et al., 2014; Shawaz et al., 2015). Psychological distress in medical students in different phases of their training has been found to be associated with symptoms of depression (Dahlin et al., 2005), anxiety (Shariati et al., 2007) burn out and even suicidal ideation (Tyssen et al., 2001) as well as a decrease in attention and learning ability (Jaykaran et al.,). These in turn, predispose to feelings of inadequacy and dissatisfaction during their clinical practice in the future, thereby having an adverse effect on the lives of the patients and health of a community at large (Shapiro et al., 2000). 
General Health Questionnaire (GHQ) is considered to be one of the best screening devices available for the identification of minor psychological disorders in the general population. It is suitable for all ages from adolescence upwards. The self-administered questionnaire focuses on two major areas, namely, the inability to carry out normal functions and the appearance of new and distressing phenomena. GHQ-12 is a quick, reliable and sensitive short form of the questionnaire having 12-items or questions. Each question is rated on a scale of $0,1,2,3$, with scores ranging from 0 to 36 . GHQ-scores 11 to 12 are typical, $>15$ implies evidence of stress and scores of more than 20 suggest psychological distress (Goldberg \& Blackwell., 1970). Previous studies have shown that approximately one third to more than half of the medical students had a higher than usual threshold score on the GHQ (Miller, 1994) with considerable variation across different years of medical study.

The Medical Student Stressor Questionnaire (MSSQ) is a newly developed instrument to identify the stressors of medical students as well as to measure the severity of stress caused by the stressors. It comprises of 40 questions representing the six stressor domains namely:

1. Academic related stressors (ARS)

2. Intrapersonal and interpersonal related stressors (IRS)

3. Teaching and learning-related stressors (TLRS)

4. Social related stressors (SRS)

5. Drive and desire related stressors (DRS)

6. Group activities related stressors (GARS)

Each question in the MSSQ rates the selfreported stress levels of the respondents on a Likert scale of 0-4 from "causing no stress at all" to "causing severe stress". Having a high score in a particular stressor domain generally indicates that the person perceives events; conditions or situations in that domain to be causing stress (Muhamad et al., 2010).

It has been postulated that the psychological health of students at the start of medical studies is similar or almost similar to their non-medical peers and tends to worsen during the medical training (Smith et al., 2007). Medical students are exposed to a new learning environment in which the vastness of syllabus, input overload and frequent examinations leave minimal opportunity for them to relax and recreate. In addition to the academic stress, they also have to deal with their emotional and physical problems while adjusting and adapting to the new environment. High expectations of the parents and peers as well as an obligation to succeed in the career, further poses a negative impact on their psychological well-being. Studies from developing countries like Malaysia (Muhamad et al., 2010), Thailand (Saipanish, 2003) and Pakistan (Mohsin et al., 2010) have reported stress among medical students during the initial formative years of medical course. However, there is very little information available in the literature about stress and its sources at the onset of medical studies especially in the Indian context.

\section{Objectives}

The present study was conducted with the aim (i) to assess the prevalence of stress in new entrants to medical undergraduate course; (ii) to determine the sources of stress and their severity in these students; (iii) to evaluate the correlation of stress with various stressors as well as demographic factors.

\section{Methods}

The present study was designed as a crosssectional, self-administered questionnaire based trial conducted in the Department of Physiology, Maulana Azad Medical College, New Delhi. The approval of the Institutional Ethical committee was obtained prior to the commencement of the study.

All the first year undergraduate medical students who were newly admitted to the Institute in the academic year 2015-16, were invited to participate in the study. The purpose of the study was fully explained to the participants and their informed signed consent was taken.

\section{Participants}

241 students out of a total of 250 new medical undergraduate students volunteered to participate in the study. Twenty seven students who returned incomplete forms were excluded from the study. The total number of participants who were finally included was 214 . The identity of each student was kept anonymous by allocating a random number to each one of them and strict confidentiality was maintained.

\section{Data collection}

Data was collected using self-reported, predesigned and pre-tested questionnaires (in English language) at the completion of their first week in the medical college. The paper-based versions of questionnaires were distributed amongst students during breaks from their teaching schedule. 
The process of filling in the questionnaire took about 15 minutes and the completed questionnaire was collected on the same day. Completion of the questionnaires was voluntary and did not affect their progression in the medical course.

The questionnaire consisted of two parts: the first part dealt with data pertaining to sociodemographic profile and personal factors such as gender, age, mother tongue, medium of education, percentage in higher secondary examination, residence of student after admission to college, family income and any kind of physical exercise done by them and for how much time.

The second part of the questionnaire comprised of the General Health Questionnaire (GHQ-12) and Medical Student Stress Questionnaire (MSSQ-40). In completing the questionnaires, the individual was required to indicate on the given Likert scale how each statement applied to them over the previous week.

\section{Measures}

\section{(i) General Health Questionnaire (GHQ-12)}

The 12-item version of the General Health Questionnaire (GHQ-12) is one of the most widely used tools to measure stress levels in the general population. The 12 items on the GHQ-12, represent 12 manifestations of stress. Respondents were asked to rate the presence of each of these manifestations in themselves' over the last week compared to their usual state on a 4-point Likert scale ('better than usual'=0, same as usual $=1$, less than usual $=2$, much less than usual $=3$ ). The possible range of scores is from 0 to 36. A total score of more than 12 was considered as a 'GHQ case' and attributed to with a higher risk of psychological morbidity (Goldberg \& Blackwell, 1970).

\section{(ii) Medical Students' Stressor Questionnaire} (MSSQ-40)

The MSSQ is a newly developed, self-report questionnaire for the identification of sources of stress and their severity in medical students. It comprises of 40 questions representing the six stressor domains namely academic (ARS), intra and interpersonal (IRS), teaching and learning (TLRS), social (SRS), drive and desire (DRS) and group activity (GARS) related stressors. Items are responded to on a Likert scale ranging from 0 (causing no stress at all) to 4 (causing severe stress). The possible range of scores is from 0 to 160 . Having a high score in a particular stressor domain generally indicates that the person perceives events; conditions or situations in that domain to be causing stress (Muhamad et al., 2010).

It can also yield the severity of stress caused by each stressor domain separately. The severity of individual stressors is calculated by summing up the item scores in that particular domain and dividing it by the total number of items in that domain. The numbers of items in various stressor domains are ARS (13), IRS and TLRS (7 each), SRS (6), DRS (3) and GARS (4). Based on the mean domain scores, the severity of various stressor domains can be graded from mild to severe stress. A mean domain score of 0-1.00 indicates that the stressor does not cause any stress or even if it does, it just causes mild stress on the participant. A mean domain score of 1.01-2.00 indicates that the stressor causes moderate stress, but the person can manage it well. A mean domain score of 2.013.00 indicates that it causes a high stress on the person leading to some emotional disturbances and mildly compromising his/her daily activities. Finally, a mean domain score of 3.01-4.00 indicates that the stressor severely causes stress and badly disturbs the emotions of the person leading to compromised daily activities due to it.

\section{Statistical analysis}

Data was analysed using Statistical Package for Social Sciences (SPSS) version 20.0 for Windows (SPSS, Inc., Chicago, Illinois, USA). All data collection forms were given serial numbers. Data were entered, checked for errors in data entry, explored and cleaned. A reliability analysis of all measures was conducted by calculating Cronbach's alpha internal consistency coefficients using the data from all participants. Any missing items not completed by the participants were replaced by median for that item. Descriptive statistics in the form of mean, standard deviation (SD) and/or range was calculated for the continuous data. Categorical data was expressed as frequency and percent. Chi-square test was used to measure association between categorical variables. Student's $t$-test for independent samples and ANOVA were used to compare the mean values of socio-demographic variables in relation to stress levels (GHQ-12), and stressors (MSSQ-40). Correlations between continuous variables were calculated using Pearson's correlation test. Logistic regression analyses were carried out to assess determinants of stressed cases. The categorized GHQ-12 score was considered the dependent variable and demographic variables and Stressor domains were considered as the independent variables. A $p$ value of $<0.05$ was considered significant for all analyses. 


\section{Results}

Demographic characteristics of the participants

Out of 250 new medical undergraduate students, 214 completed and returned the questionnaires giving an overall response rate of $85.6 \%$. Their mean age was $18.50 \pm 1.07$ years with a range of 17-22 years. One hundred and thirty students were male $(60.7 \%)$ and 84 were female $(38.8 \%)$. Mother-tongue of majority of the participants was Hindi $(89.3 \%)$ and medium of education in school was English $(97.2 \%)$. More than half of participants $(50.9 \%)$ had scored above $90 \%$ in their higher secondary examinations. Only $27.1 \%$ of the participants were hostellers while rest were all day-scholars. More than one third participants $(38.3 \%)$ did some form of regular physical exercise. The family income of almost half $(47.7 \%)$ of the participants was $>1$ lakh rupees per month (Table 1).

Stress and sources of stress: GHQ-12, MSSQ40 and its domains.

The internal reliability (Cronbach's alpha) for GHQ-12 and MSSQ-40 were 0.83 and 0.94 respectively. The mean (SD) scores for GHQ, MSSQ and various stressor domains are shown in Table 2.

Table 1: Demographic characteristics of the participants $(n=214)$

\begin{tabular}{|c|c|c|c|}
\hline \multicolumn{2}{|c|}{ Studied Variables } & \multirow{2}{*}{$\begin{array}{r}\text { Frequency } \\
130 \\
84\end{array}$} & \multirow{2}{*}{$\begin{array}{r}\text { Percent } \\
60.7 \\
38.8\end{array}$} \\
\hline Gender & $\begin{array}{l}\text { Male } \\
\text { Female }\end{array}$ & & \\
\hline Mother-Tongue & $\begin{array}{l}\text { Hindi } \\
\text { English } \\
\text { Others }\end{array}$ & $\begin{array}{r}191 \\
2 \\
21\end{array}$ & $\begin{array}{r}89.3 \\
0.9 \\
21.0\end{array}$ \\
\hline Medium of Education & $\begin{array}{l}\text { Hindi } \\
\text { English } \\
\text { Others }\end{array}$ & $\begin{array}{r}4 \\
208 \\
2\end{array}$ & $\begin{array}{r}1.9 \\
97.2 \\
0.9\end{array}$ \\
\hline Percent in HSE\# & $\begin{array}{l}\leq 50 \\
51-75 \\
76-90 \\
\geq 91\end{array}$ & $\begin{array}{r}1 \\
19 \\
81 \\
109\end{array}$ & $\begin{array}{r}0.5 \\
8.9 \\
37.9 \\
50.9\end{array}$ \\
\hline Stay & $\begin{array}{l}\text { Day-scholar } \\
\text { Hosteler }\end{array}$ & $\begin{array}{r}156 \\
58\end{array}$ & $\begin{array}{l}72.9 \\
27.1\end{array}$ \\
\hline Exercise & $\begin{array}{l}\text { Yes } \\
\text { No }\end{array}$ & $\begin{array}{r}82 \\
132\end{array}$ & $\begin{array}{l}61.7 \\
38.3\end{array}$ \\
\hline $\begin{array}{l}\text { Family Income (Indian } \\
\text { Rupees/month) }\end{array}$ & $\begin{array}{l}<20000 \\
21000-50000 \\
51000-100000\end{array}$ & $\begin{array}{l}21 \\
47 \\
45\end{array}$ & $\begin{array}{r}9.8 \\
21.9 \\
21.0\end{array}$ \\
\hline
\end{tabular}

Table 2: Descriptives and internal consistencies for both questionnaires $(n=214)$

\begin{tabular}{lcrc}
\hline \multirow{2}{*}{$\begin{array}{c}\text { Questionnaire } \\
\text { (number of items) }\end{array}$} & \multicolumn{3}{c}{ Current Sample } \\
\cline { 2 - 4 } & $\begin{array}{c}\text { Sample range } \\
\text { (Questionnaire Range) }\end{array}$ & Mean (SD) & $\boldsymbol{\alpha}$ \\
\hline GHQ-12 (12) & $0-34(0-36)$ & $11.93(5.60)$ & 0.83 \\
MSSQ (40) & $0-136(0-160)$ & $52.09(25.11)$ & 0.94 \\
ARS (13) & $0-47(0-52)$ & $21.83(9.76)$ & 0.86 \\
IRS (7) & $0-23(0-28)$ & $7.94(5.80)$ & 0.83 \\
TLRS (7) & $0-26(0-28)$ & $8.42(5.27)$ & 0.81 \\
SRS (6) & $0-23(0-24)$ & $6.25(3.52)$ & 0.53 \\
DRS (3) & $0-11(0-12)$ & $2.48(2.55)$ & 0.66 \\
GARS (4) & $0-15(0-16)$ & $5.17(3.38)$ & 0.73 \\
\hline
\end{tabular}

GHQ-12 = General Health Questionnaire, MSSQ = Medical Student Stressor Questionnaire, ARS = Academic Related Stressors, IRS = Inter and Intrapersonal Related Stressors, TLRS = Teaching and Learning Related Stressors, SRS = Social Related Stressors, DRS = Drive and Desire Related Stressors, GARS = Group Activities Related Stressors 
The overall prevalence of stress (GHQ score of 12 as the cut-off point) among the students was $42.1 \%$ out of which almost $8 \%$ had GHQ scores of more than 20 (Figure 1).

Severity of academically related stressors was found to be the highest among the medical students, with more than one third $(34.1 \%)$ of the participants perceiving them to be causing high or severe stress. In all other domains, mild to moderate degrees of stress was predominantly reported by more than $85 \%$ of the participants. Social related stressors were found to be the least stressful with only $4.7 \%$ of the participants perceiving them to be causing high or severe stress (figure 2a-2f).

Self-rated severity of all except intrapersonal and interpersonal related stressors was found to be significantly higher in stressed $(p<0.05$ to $p<0.01$ ) as compared to the non-stressed participants. Figures 2a-f shows the selfreported severity of various stressors on the basis of prevalence of stress.

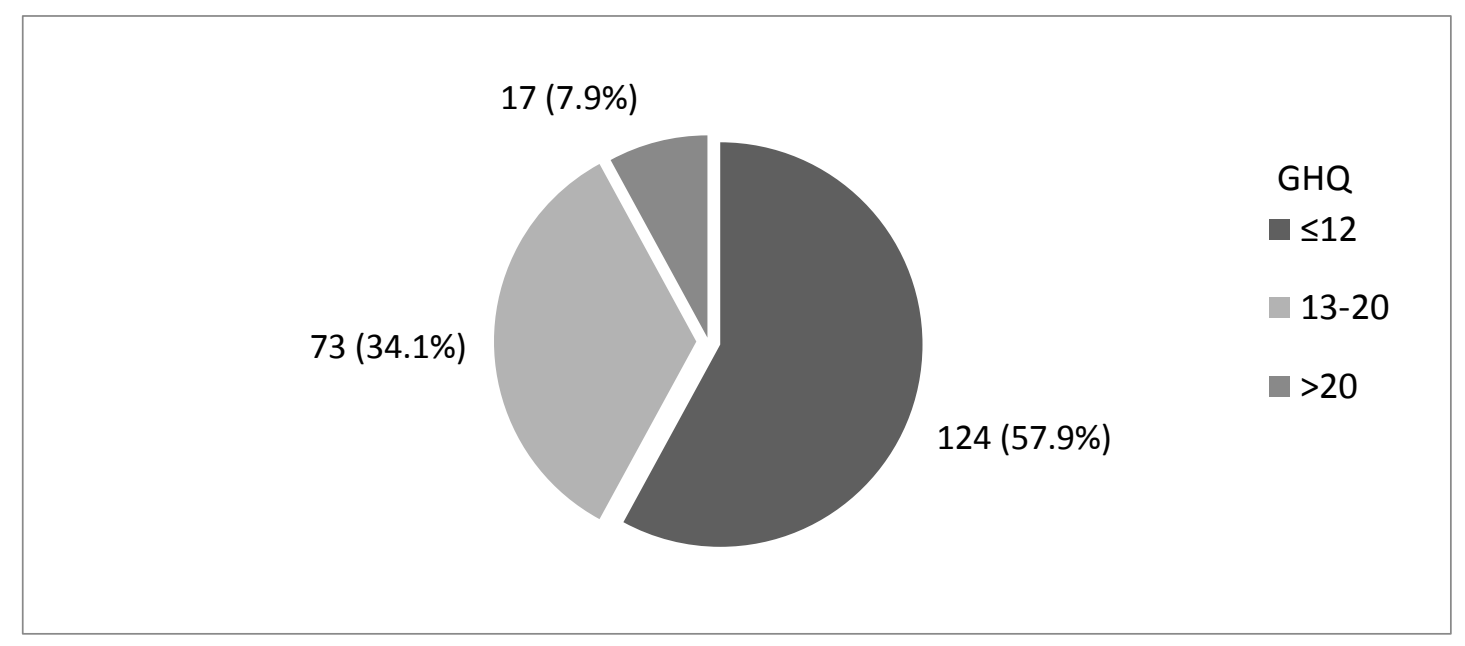

Values are frequency (percent) for each cut-off point

Figure 1: Prevalence of stress $(n=214)$

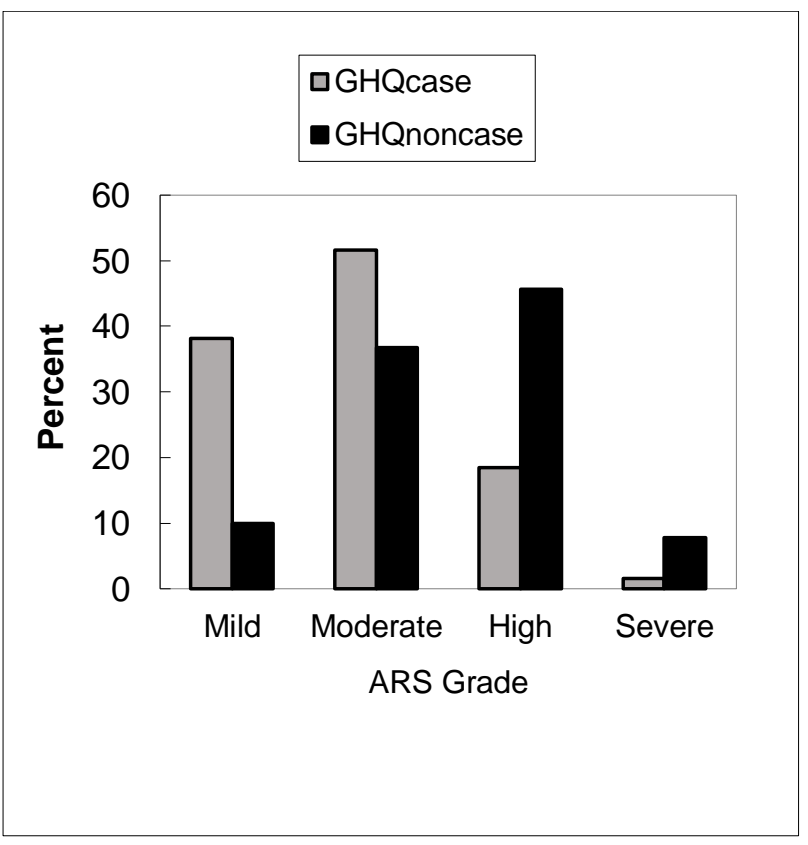

(a)

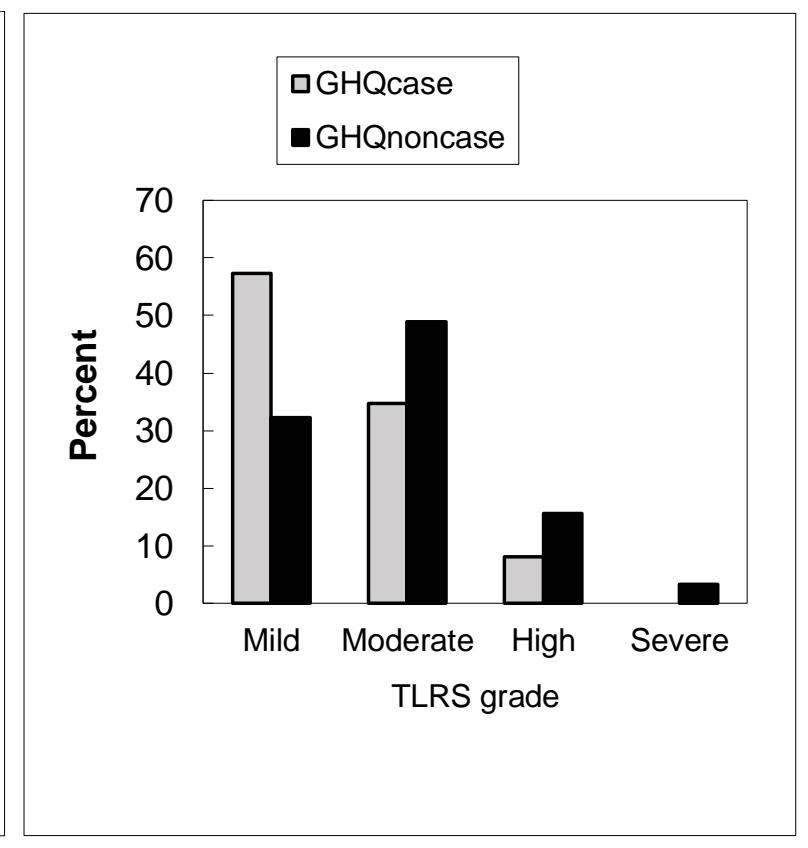

(b)

Figure 2: Self-rated severity of stressors

$A R S g d=$ Severity grade of academic related stressors, IRSgd = Severity grade of Inter and Intrapersonal related stressors, TLRSgd = Severity grade of Teaching and Learning related stressors, SRSgd = Severity grade of Social related stressors, DRSgd = Severity grade of Drive and Desire related stressors, GARSgd = Severity grade of Group Activities related stressors 


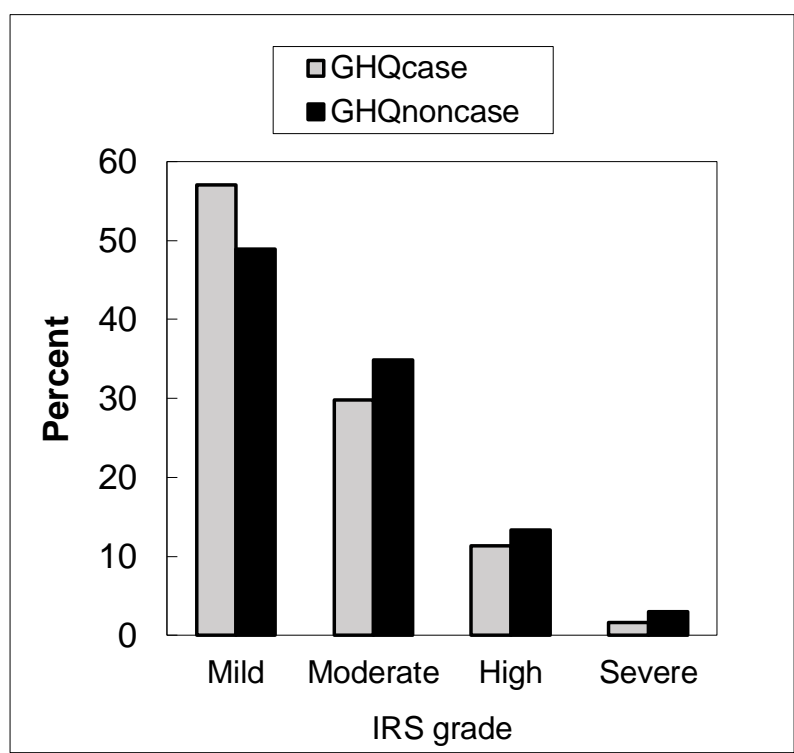

(c)

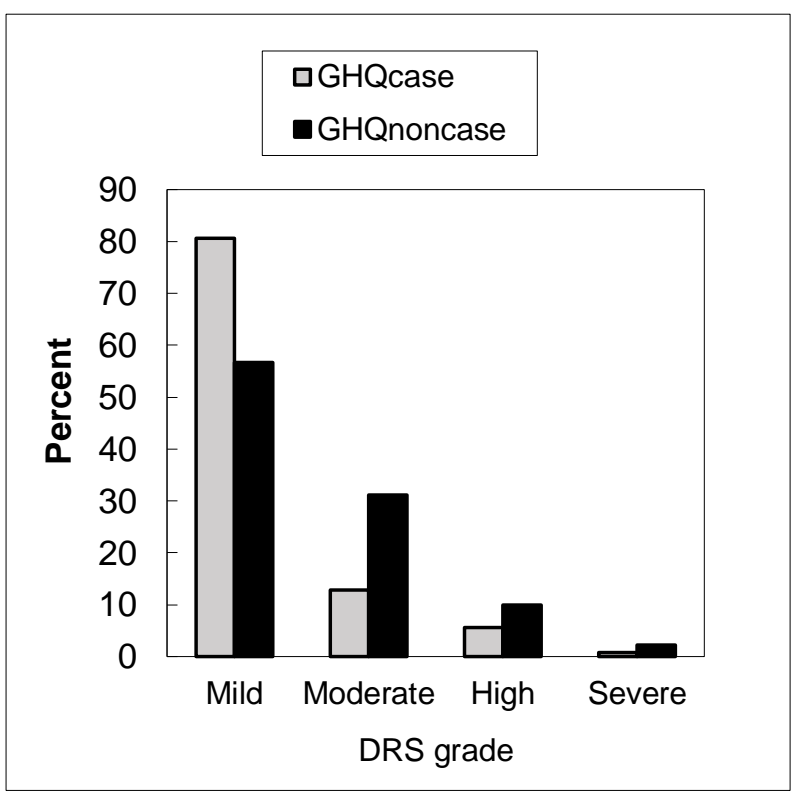

(e)

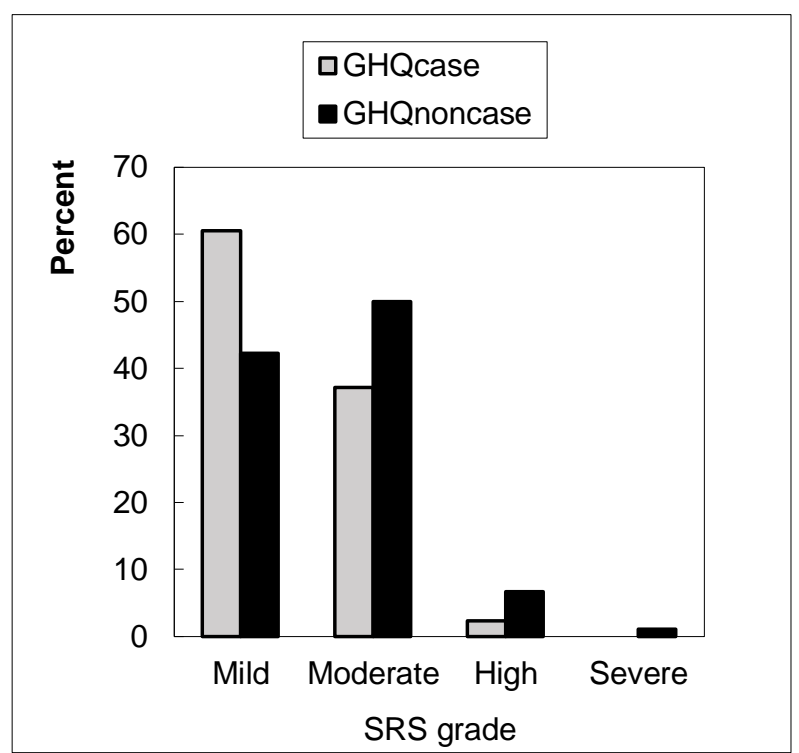

(d)

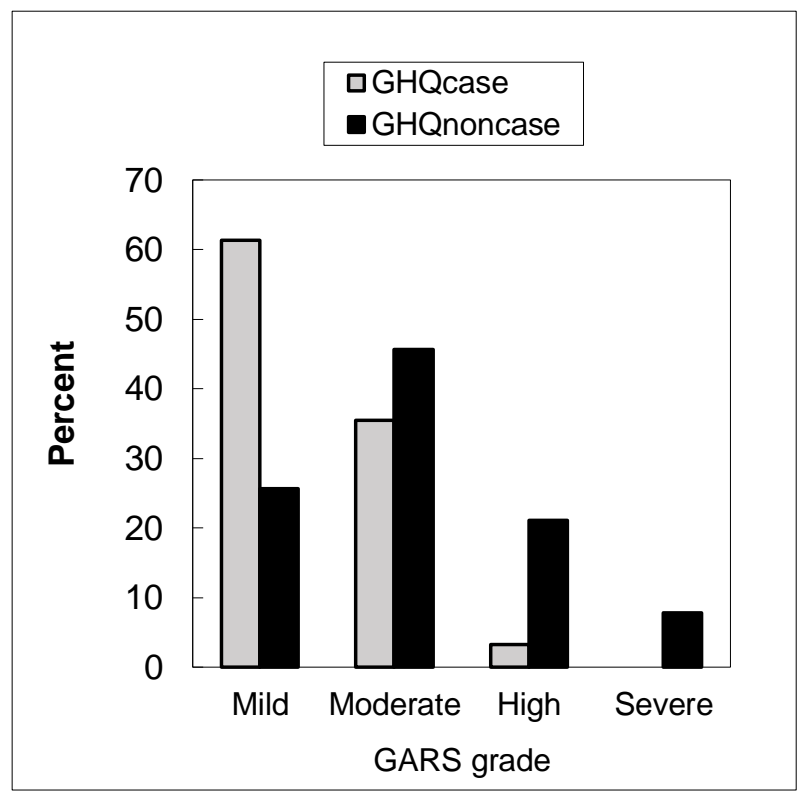

(f)

Figure 2 (continued): Self-rated severity of stressors

$A R S g d=$ Severity grade of academic related stressors, IRSgd = Severity grade of Inter and Intrapersonal related stressors, TLRSgd = Severity grade of Teaching and Learning related stressors, SRSgd = Severity grade of Social related stressors, DRSgd = Severity grade of Drive and Desire related stressors, GARSgd = Severity grade of Group Activities related stressors

\section{Correlations}

There was no difference in the prevalence of stress on the basis of gender, mother tongue, medium of education, percentage of marks in HSE, place of stay, family income or exercise. There was a significant correlation of female gender with both ARS and TLRS $(r=0.17$ and 0.14 respectively, $p<0.05$ ). Higher levels of GHQ were significantly correlated with total MSSQ scores $(r=0.48, p<0.01)$, as well as all the six domains of MSSQ ( $r=0.22$ to 0.53 , $\mathrm{p}<0.01)$.

By logistic regression analysis, stressed cases were found to be associated with only GARS $(B=1.095, \quad S E=0.31$, Wald=12.39, $p<0.001)$. Stressed cases did not show any association with gender, mother tongue, medium of education, percentage of marks in HSE, place of stay, family income, exercise or other stressor domains $\left[X^{2}(8, n=214)=7.133, p=0.52\right]$. 


\section{Discussion}

The key findings of our research are the high prevalence of stress in the new entrants to medical undergraduate course as assessed by the GHQ-12 with no significant difference between male and female participants. Academic related stressors were reportedly more common both in terms of frequency of occurrence and severity. Self-rated severity of all except intrapersonal and interpersonal related stressors was found to be significantly higher in stressed as compared to the nonstressed participants. GHQ scores showed significant correlation with the overall MSSQ scores as well as to all its stressor domains. However, stressed cases were found to be associated with only GARS. Significant association was not observed between stressed cases and other stressors or any of the demographic correlates.

The experimental design of the present study is different from those of earlier studies where the prevalence of stress was either evaluated in the students after completion of at least two months in the medical college (Muhamad et al., 2010) or in students who were at different stages of their medical training (Mohsin et al., 2010; Najmeh et al., 2012; Chauhan et al., 2014). In our study we assessed the prevalence of stress in new entrants to medical undergraduate course who had completed their first week in the college. We chose this period to avoid the timings of stressful examinations, which could have introduced potential measurement bias. Moreover, it has been postulated here that the psychological health of students at the start of medical studies is similar or almost similar to their non-medical peers and tends to worsen during the course of medical training (Smith et al., 2007). Thus, we reasoned that the selfreported stress levels would reflect the natural levels of stress in these students.

The relatively high response rate seen in this study is in concurrence with various other previous studies (Mohsin et al., 2010; Muhamad et al., 2010). Considering the fact that stress is becoming an emerging issue amongst medical students across the globe, this response rate indicates the growing perceptive concern of the budding medicos towards their psychological wellbeing even at the commencement of the professional course.

In the present study, both GHQ-12 and MSSQ40 questionnaires had high reliability in the participants with Cronbach's alpha value of 0.83 and 0.94 respectively. Reliability analysis of various stressor domains of MSSQ also showed that, except SRS (Cronbach's alpha=0.53), all the other five domains were reliable and had a high internal consistency as their Cronbach's alpha coefficient value ranged from 0.66-0.86, which is more than the acceptable cut-off point of 0.6 (Downing, 2004).

Our GHQ-12 data illustrated a high prevalence of stress amongst fresh medical undergraduate students of our institute with almost $8 \%$ of the students self-reporting to be severely stressed. These observations in our study are in concurrence with various other previous studies that have assessed stress levels in medical students using different study populations and protocols (Sherina et al., 2003; Zaid et al., 2007; El-Gilany et al., 2008; Najmeh et al., 2012). However, it is noteworthy that the overall $42.1 \%$ prevalence of stress observed in our study is much lower than several other studies conducted in India (Harlina et al., 2014) and abroad (Guthrie et al., 1995; Saipinish, 2003; Sani et al., 2012). One possible reason for the relatively low stress prevalence in our students could be that they had just entered the course one week prior and may have still been experiencing the stages of novelty and euphoria. In addition, during this time, they had yet to face difficult subjects because most subjects studied during the initial months are ones they have learned during secondary schooling. Furthermore, the stress prevalence in our study was comparable in male and female students with no significant difference found on the basis of either gender or any other socio-demographic factor. These results are in contradiction to some earlier work that had reported significantly higher levels of stress prevalence in female students as compared to their male counterparts (Mohsin et al., 2010; Sani et al., 2012). The disparity in the methodological factors relating to the measures used or subject selection (students in different years of medical training) may have been potential sources for the inconsistency in our findings with those of previous research.

In the present study, we observed that the severity of academically related stressors was highest among the medical students with more than one third $(34.1 \%)$ of the participants perceiving them to be causing high or severe stress. This was surprising considering the fact that the participants had only completed their first week in the college. Since ours is amongst one of the premiere medical institutes of the country, the students selected here are also from amongst the national and state toppers. Being over achievers and perfectionists, these students have high ambitions also. It is perhaps their high self-expectation and desire to excel 
as medical professional that could have contributed to their perceiving ARS as causing severe stress. Another plausible cause could be the realization that medical training and curriculum required learning and mastering a huge amount of knowledge, attitude and skills in limited time frame.

Our MSSQ data further illustrated that, in all other stressor domains, mild to moderate degrees of stress was predominantly reported by more than $85 \%$ of the participants. SRS were found to be the least stressful with less than $5 \%$ of the participants perceiving them to be causing high or severe stress. Self-rated severity of all except IRS was found to be significantly higher in stressed $(p<0.05$ $p<0.01)$ as compared to the non-stressed participants. There was a significant correlation $(p<0.05)$ of female gender with both ARS and TLRS. Higher levels of GHQ were significantly correlated $(p<0.01)$ with total MSSQ scores as well as all the six stressor domains of MSSQ.

The overall pattern of stressors in our study is more or less in conformity with the findings of studies done in other medical schools, where investigators report that the majority of the stressors were primarily related to academic matters (Coburn \& Jovaisas, 1975; Supe, 1998; Shariati et al., 2007). However, the frequency of some stressors may be significantly different from studies done elsewhere (Kaufmann et al., 1998). For instance, earlier works have reported that approximately half of the respondents rated intra-interpersonal related stressor or psychosocial and emotional factors to be causing severe stress (Baldwin et al., 1991; Sreeremareddy, 2007; Harlina et al., 2014). In a few other studies, respondents were found to be dissatisfied with quality of education imparted and rated teaching and learning related stressors to be causing severe stress (Michie, 2002; Shariati et al., 2007; Brown et al., 2009). These researchers reasoned that medical training was highly stressful as the students besides pursuing knowledge in medical university, also had to simultaneously adjust and adapt to the changing of education system, lifestyle and social environment. Approximately three-fourths of the participants in our study hailed from similar sociodemographic background and almost all $(97.2 \%)$ of them have had their schooling English medium. This might have led to the lower severity of stress reported by our participants in all other stressor domains except ARS. GARS relates to participation in group discussions, group presentations and others expectations to do well. Some educational activities in basic science studies also involves group activities. Logistic regression analysis in the present study revealed significant association $(p<0.001)$ of stressed cases with only GARS suggesting that the students were having difficulty with group activities.

The high prevalence of stress seen among medical students is a cause of concern as it may impair the behaviour of students, decrease psychological health, diminish learning, and ultimately affect patient care after completing medical training. Studies done on first year medical students in different medical schools have further shown that those who perceived academic related stressors as causing high to severe stress had seven to sixteen times higher risk of developing psychological distress in future compared to those who perceived them as causing mild to moderate stress (Kaufmann et al., 1998). In the present study too, academic related factors contributed relatively more in causing stress compared to other stressor domains. Moreover, previous studies also suggest that mental health worsens after students join medical school and remains poor throughout the course (Dyrbye et al., 2005), especially in the transition from basic science teaching to clinical training (Helmers et al., 1997). As the study findings showed a high prevalence of stress among new entrants, we suggest supporting and training them properly in order to avoid stress burnout. This will also help them cope well with stress in later years. Furthermore, it is important to detect stressed students early to prevent deleterious long term effects of stress on students.

\section{Conclusion}

The present study reports the prevalence and sources of stress in fresh entrants to the medical undergraduate course. It also grades the severity of various stressor domains in these subjects. It revealed that academic related factors were greater sources of stress as compared to non-academic factors. To the best of our knowledge, the current data are one of the first to measure the sources of stress and their severity in this population.

\section{Limitations}

Since the information was collected on selfadministered questionnaires/instruments we cannot rule out information bias. Crosssectional design of our study is yet another limitation. Prospective studies are necessary to study the associations between prevalence of stress and stressors. 


\section{References}

Baldwin Jr, D.C., Daugherty, S.R. \& Edward, J. Eckenfels. (1991) Student perceptions of mistreatment and harassment during medical school - a survey of 10 United States schools, Western Journal of Medicine, 155, 2, pp.140-145.

Brown, S.D., Goske, M.J. \& Johnson, C.M. (2009) Beyond substance abuse: stress, burnout, and depression as causes of physician impairment and disruptive behaviour, Journal of American College of Radiology, 6, 7, pp.479-485.

Chauhan, H.M., Shahb, H.R., Chauhan, S.H. \& Chaudhary, S.M. (2014) Stress in medical students: a cross sectional study, International Journal of Biomedical \& Advance Research, 5, 6, pp. 292-294.

Coburn, D. \& Jovaisas, A.V. (1975) Perceived sources of stress among first year medical students, Medical Education, 50, 6, pp. 589-95.

Dahlin, M., Joneborg, N. \& Runeson, B. (2005) Stress and depression among medical students: a cross-sectional study, Medical Education, 39, 6, pp. 594-604.

Downing, S.M. (2004) Reliability: on the reproducibility of assessment data, Medical Education, 38, 9, pp.1006-1012.

Dyrbye, L., Thomas, M.R. \& Shanafelt, T.D. (2005) Medical student distress: causes, consequences, and proposed solutions, Mayo Clinic Proceedings, 80, 12, pp.1613-1622.

El-Gilany, A.H., Amr. M. \& Hammad, S. (2008) Perceived stress among male students in Egypt \& Saudi Arabia: effect of sociodemographic factors, Annals of Saudi Medicine, 28, 6, pp. 442-448.

Goldberg, D.P. \& Blackwell, B. (1970) Psychiatric illness in general practice. A detailed study using a new method of case identification. British Medical Journal, 23, 1, pp. 439-443.

Guthrie, E.A., Black, D., Shaw, C.M., Hamilton, J. \& Creed, F.H. (1995) Embarking upon a medical career: psychological morbidity in first year medical students, Medical Education, 29, 5, pp. 337-341.

Harlina, H.S., Salam, A., Roslan, R., Hasan, N.A., Jin, T.H. \& Othman, M.N. (2014) Stress and its association with the academic performance of undergraduate fourth year medical students at Universiti Kebangsaan Malaysia, International Medical Journal Malaysia, 13, 1, pp.17-22.

Helmers, K.F., Danoff, D., Steinert, Y., Leyton, M. \& Young, S.N. (1997) Stress and depressed mood in medical students, law students, and graduate students at McGill University. Academic Medicine, 72, 8, pp. 708-714.
Jaykaran, Yadav, P., Bharadwaj, P., Panwar, A. \& Chavda, N. (2009) Perception of faculties regarding the stress in medical education-a qualitative study, The Internet Journal of Epidemiology, 7, 1.

Kaufman, D.M., Day, V. \& Mensink, D. (1998) Stressors in Medical School: Relation to curriculum format and year of study, Teaching and Learning in Medicine, 10, 3, pp. 188-194.

Lazarus, R.S. (1990) Theory Based Stress Measurement, Psychological Inquiry, 1, 1, pp.313.

Mannapur, B., Dorle, A.S. \& Hiremath, I.D. (2010) A study of psychological stress in undergraduate medical students at SN Medical College, Bagalkot, Karnataka, Journal of Clinical and Diagnostic Research, 4 4, pp. 2869-2874.

Michie, S. (2002) Causes and management of stress at work, Occupational \& Environmental Medicine, 59, 1, pp. 67-72.

Miller, P.M. (1994) The first year at medical school: some findings and student perceptions, Medical Education, 28, 1, pp. 5-7.

Mohsin, S. Hassan, S., Malik, S. \& Sreeramaredd, C.T. (2010) Perceived stress, sources and severity of stress among medical undergraduates in a Pakistani medical school, BMC Medical Education, 10, 2, pp. 1-8.

Muhamad, S.B.Y., Ahmad, F.A.R. \& Mohd, J.Y. (2010) Prevalence and sources of stress among medical students in University Sains Malaysia, The Malaysian Journal of Medical Sciences, 17, 1, pp.30-37.

Najmeh, J. Amir, L. \& Ali. M. (2012) Mental health of medical students in different levels of training, International Journal of Preventive Medicine, 3, 1, pp. S107-S112.

Saipanish, R. (2003) Stress among medical students in a Thai Medical School: medical teacher, An International Journal of Education in Health Sciences, 25, 5, pp. 502-506.

Sani, M., Mahfouz, M.S., Bani, I., Alsomily, A.H., Alagi, D., Alsomily, N.Y., Madkhaly, F.M., Madkhali, R., Hakami, A.A.M., Hakami, A., Shaabi, S., Al Ebrahim, S., Mashiakhi, S.H., Ageel, B. \& Asiri, S. (2012) Prevalence of stress among medical students in Jizan University, Kingdom of Saudi Arabia, Gulf Medical Journal, 1, pp.19-25.

Shapiro, S.L., Shapiro, D.E. \& Schwartz, G.E. (2000) Stress management in medical education: a review of the literature. Journal of the Association of American Medical College, 75, 7, pp. 748-759. 
Shariati, M., Yunesian, M. \& Vash, J.H. (2007) Mental health of medical students: a crosssectional study in Tehran, Psychological Reports, 100, 2, pp. 346-354.

Shawaz, I., Sandhya, G. \& Venkatarao, E. (2015) Stress, anxiety \& depression among medical undergraduate students \& their sociodemographic correlates, Indian Journal of Medical Research, 141, 3, pp. 354-357.

Sherina, M.S., Lekhraj, R. \& Nandarajan. K. (2003) Prevalence of Emotional Disorder among Medical Students in a Malaysian University. Asia Pacific Family Medicine, 2,4, pp. 213-217.

Smith, C.K., Peterson, D.F., Degenhardt, B. \& Johnson, J.C. (2007) Depression, anxiety and perceived hassles among medical students. Psychology, Health\& Medicine, 12, 1, pp.31-39.
Sreeramareddy, C.T., Shankar, P.R., Binu, V.S., Mukhopadhyay, C., Ray, B. \& Menezes, R.G. (2007) Psychological morbidity, sources of stress and coping strategies among undergraduate medical students of Nepal. BioMed Central Medical Education, 2, 7, pp.26.

Supe, A.N. (1998) A study of stress in medical students at Seth G.S. Medical College. Journal of Postgraduate Medicine, 44, pp. 1-6.

Trushna, S., Geetanjali, P.M. \& Shah, G.V. (2014) Stress and stressors in medical education and evaluation of different modalities to overcome it, Scholars Journal of Applied Medical Sciences, 2, 3, pp.1123-1126. 